EPJ manuscript No.

(will be inserted by the editor)

\title{
Plotting the Differences Between Data and Expectation
}

\author{
Georgios Choudalakis ${ }^{1}$ and Diego Casadei ${ }^{2}$ \\ 1 University of Chicago, Enrico Fermi Institute, 5640 South Ellis Avenue, Chicago, IL-60637, USA \\ e-mail: gchouda@alum.mit.edu \\ 2 Department of Physics, New York University, 4 Washington Place, New York, NY-10003, USA \\ e-mail: diego.casadei@cern.ch
}

the date of receipt and acceptance should be inserted later

\begin{abstract}
This article proposes a way to improve the presentation of histograms where data are compared to expectation. Sometimes, it is difficult to judge by eye whether the difference between the bin content and the theoretical expectation (provided by either a fitting function or another histogram) is just due to statistical fluctuations. More importantly, there could be statistically significant deviations which are completely invisible in the plot. We propose to add a small inset at the bottom of the plot, in which the statistical significance of the deviation observed in each bin is shown. Even though the numerical routines which we developed have only illustration purposes, it comes out that they are based on formulae which could be used to perform statistical inference in a proper way. An implementation of our computation is available at: https://github.com/dcasadei/psde.
\end{abstract}

PACS. 02.70.Rr General statistical methods

\section{Introduction}

Most analyses compare the observed data to the expectation resulting from a theoretical model, like the Standard Model (SM), or some other hypothesis, like a best fitting function, or a Monte Carlo simulated distribution. It is common to make histograms in logarithmic scale, because their contents span orders of magnitude. Since differences are difficult to see in a logarithmic scale, an inset plot is often made at the bottom of the histogram. In this article, we focus on this inset and propose a way to make it intuitive and accurate. By "intuitive" we mean that it should make it obvious which bins contain an excess of data and which contain a deficit, while significant deviations should look more striking than insignificant ones. By "accurate" we mean that it should represent the actual significance of the deviation in each bin, rather than some approximation.

Below we define statistical significance in two probability models, Poisson and binomial. In Section 2 we show a sequence of presentation options that could be used (and most of them have been used), explaining their strengths and limitations. This will motivate, through a series of incremental improvements, our final proposal, which is given in Section 2.4. Finally, if the expectation is only known within some uncertainty, this uncertainty can be taken into account in the comparison to the data, as explained in Section 3. In this paper, we use the ROOT framework [1] to produce the plots and provide suggestions for implementing our formulae with this widely used software for data analysis and visualization, although our recommendations can be easily implemented within other frameworks too.

\subsection{Definition of statistical significance}

The key notion, which is needed to define the statistical significance, is the probability of finding a deviation at least as big as the one observed in the data, under the assumption that the chosen theoretical model describes our system. This probability is commonly called the " $p$-value" and it usually spans several orders of magnitude. For this reason, it is convenient to translate the $p$-value into a " $z$-value", which is the deviation at the right of the mean of a Gaussian distribution, expressed in units of standard deviations, which would correspond to the same $p$-value. The equation which maps each $p$-value to a $z$-value, and vice versa, is:

$$
p \text {-value }=\int_{z \text {-value }}^{\infty} \frac{1}{\sqrt{2 \pi}} e^{-\frac{x^{2}}{2}} d x,
$$




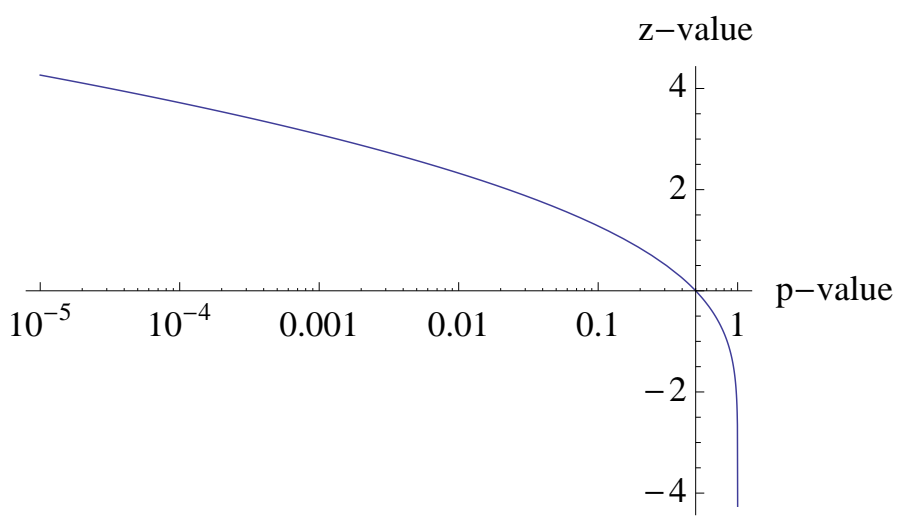

Fig. 1. Relationship between $p$-value and $z$-value.

which is shown in Fig. 1. In ROOT, the $z$-value can be computed in one line of code, using the inverse error function:

$\operatorname{sqrt}(2)$.$* TMath :: \operatorname{Erf} \operatorname{Inverse}(1 .-2 . *$ pvalue $)$

A $z$-value $\geq 0$ corresponds to a $p$-value $\leq 0.5$, and negative $z$-values correspond to $p$-value $>0.5$. Significant deviations are characterized by quite small $p$-values, corresponding to $z$-values $\geq 3$. For example, it is common to refer to a $p$-value $=2.87 \times 10^{-7}$ as a " $5 \sigma$ effect", meaning that the corresponding $z$-value is 5 . Such deviations are usually considered very significant and a $z$-value of 5 or more is conventionally required in High Energy Physics to claim a discovery [2]. On the other hand, a $z$-value which is less than 1-2 units represents a common statistical fluctuation, which is uninteresting. The fluctuations are even more probable (i.e. less interesting) when the $z$-value is negative.

The $p$-value depends on the statistical distribution that the data are supposed to follow, the so-called "probability model". The cases of Poisson and binomial distributed data are most common in experimental physics, and are addressed in this note.

\subsection{Poisson model}

This is the most common case, where event counts are plotted in each bin and the number of entries in each bin follows a Poisson distribution (e.g. Ref. [3]). If $B \in \mathbb{R}$ events are expected in a bin, the probability of observing $D \in \mathbb{N}$ events is

$$
P(D \mid B)=\operatorname{Poi}(D \mid B)=\frac{B^{D}}{D !} e^{-B}
$$

( $B$ is also the variance of $D)$. The Poisson $p$-value is

$$
p \text {-value }= \begin{cases}\sum_{n=D}^{\infty} \frac{B^{n}}{n !} e^{-B}=1-\sum_{n=0}^{D-1} \frac{B^{n}}{n !} e^{-B} & , D>B \\ \sum_{n=0}^{D} \frac{B^{n}}{n !} e^{-B} & , D \leq B\end{cases}
$$

The above sums are simplified thanks to the identity:

$$
\sum_{n=0}^{D-1} \frac{B^{n}}{n !} e^{-B}=\frac{\Gamma(D, B)}{\Gamma(D)},
$$

where

$$
\begin{aligned}
\Gamma(D, B) & =\int_{B}^{\infty} t^{D-1} e^{-t} d t \\
\Gamma(D) & =\int_{0}^{\infty} t^{D-1} e^{-t} d t .
\end{aligned}
$$


The ratio between the upper incomplete Gamma function $\Gamma(s, x)$ and the Gamma function $\Gamma(s)$ in the previous equation is known as the upper regularized Gamma function

$$
Q(s, x)=\frac{\Gamma(s, x)}{\Gamma(s)}=1-P(s, x)
$$

where $P(s, x)$ is the cumulative distribution function for Gamma random variables with shape parameter $s$ and scale parameter 1 [4]. In ROOT this function is available as

$$
Q(s, x)=\text { ROOT : :Math : : inc_gamma_c }(\mathrm{s}, \mathrm{x}),
$$

such that

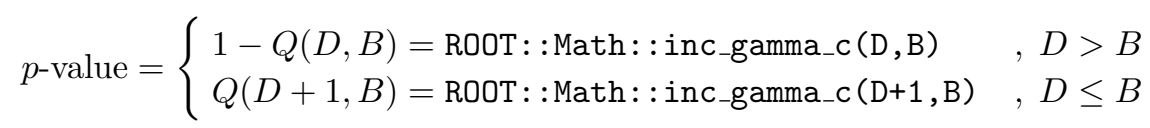

One should notice that it is not always true that the bin population follows the Poisson distribution. This does not happen when the total number of entries in a histogram is not a random variable, in which case the events in each bin are distributed accordingly to the multinomial distribution, which means that the bins cannot be considered statistically independent as we assumed in this section. In the extreme case of a fixed total number of entries and only two bins, the content of each bin follows the binomial distribution: if there is an excess of counts in one bin, with respect to the expectation, then the other bin must have a deficit in order to preserve the total number of events.

\subsection{Binomial model}

If the plotted quantity is a Bernoulli success rate, then it follows a binomial distribution. A common example would be a trigger efficiency "turn-on curve" like Fig. 1 in Ref. [6], where it would be informative to show how significantly the observed trigger rate differs from the simulation. Another example is $F_{\chi}\left(m_{j j}\right)$ in Ref. [5]: if this event rate were significantly different than the SM prediction, this could be a sign of certain new physics.

Let us define the following notation:

$n \in \mathbb{N}$ : the number of events initially observed in a bin, a subset of which will survive the selection; $k_{\text {obs }} \in\{0,1, \ldots, n\}:$ the observed number of selected events; $\varepsilon \in \mathbb{R}$ : the expected success rate, i.e. the selection efficiency, to which we compare the observed success rate $k_{\text {obs }} / n$.

The binomial distribution gives the probability of observing $k$ events passing the selection out of the initial $n$ events:

$$
P(k \mid n, \varepsilon)=\operatorname{Bi}(k \mid n, \varepsilon)=\left(\begin{array}{l}
n \\
k
\end{array}\right) \varepsilon^{k}(1-\varepsilon)^{n-k}
$$

The expected number of surviving events is $E[k \mid n, \varepsilon]=n \varepsilon$, with variance $V[k \mid n, \varepsilon]=n \varepsilon(1-\varepsilon)$. Finally, the Binomial $p$-value is defined as

$$
p \text {-value }= \begin{cases}\sum_{n=k_{\text {obs }}}^{n}\left(\begin{array}{l}
n \\
k
\end{array}\right) \varepsilon^{k}(1-\varepsilon)^{n-k} & , k_{\text {obs }} \geq n \varepsilon \\
k_{\text {obs }}\left(\begin{array}{l}
n \\
k
\end{array}\right) \varepsilon^{k}(1-\varepsilon)^{n-k} & , k_{\text {obs }}<n \varepsilon\end{cases}
$$

The cumulative distribution function of the binomial model can be represented in terms of the regularized incomplete Beta function [4] $I_{x}(a, b)=\int_{0}^{x} t^{a-1}(1-t)^{b-1} \mathrm{~d} t / B(a, b)$ where the Euler's Beta function can be represented in terms of the Gamma function as $B(a, b)=\Gamma(a) \Gamma(b) / \Gamma(a+b)$. The regularized incomplete beta function is available in ROOT as

$$
I_{x}(a, b)=\text { ROOT }: \text { Math }:: \text { inc_beta }(\mathrm{x}, \mathrm{a}, \mathrm{b}),
$$

which makes it possible to implement what follows.

Let us start with the deficit case $k_{\mathrm{obs}}<n \varepsilon$, for which the $p$-value coincides with the cumulative distribution function:

$$
p \text {-value }=P\left(k \leq k_{\text {obs }}\right)=I_{1-\varepsilon}\left(n-k_{\text {obs }}, k_{\text {obs }}+1\right), \quad k_{\text {obs }}<n \varepsilon .
$$

For the case of an excess, we use two identities. The first is $I_{x}(a, b)=1-I_{1-x}(b, a)$ and the second comes directly from the binomial distribution and is the starting point of the following chain of equations, whereas the previous identity is used in the last step:

$$
P\left(k \geq k_{\mathrm{obs}}+1\right)=1-P\left(k \leq k_{\mathrm{obs}}\right)=1-I_{1-\varepsilon}\left(n-k_{\mathrm{obs}}, k_{\mathrm{obs}}+1\right)=I_{\varepsilon}\left(k_{\mathrm{obs}}+1, n-k_{\mathrm{obs}}\right)
$$




\begin{tabular}{c|rl||r|rl} 
Bin number & Observed & Expected & Bin number & Observed & Expected \\
\hline \hline 1 & 121687 & 121688 & 21 & 233 & 226.509 \\
2 & 222014 & 221422 & 22 & 129 & 144.086 \\
3 & 223741 & 223832 & 23 & 90 & 91.4576 \\
4 & 189486 & 190065 & 24 & 51 & 57.9372 \\
5 & 148673 & 148218 & 25 & 53 & 36.6361 \\
6 & 109932 & 109876 & 26 & 20 & 23.1279 \\
7 & 79553 & 78760.1 & 27 & 15 & 14.5779 \\
8 & 56420 & 55119.7 & 28 & 13 & 9.17558 \\
9 & 38771 & 37889.4 & 29 & 3 & 5.76764 \\
10 & 25628 & 25684.7 & 30 & 4 & 3.621 \\
11 & 17309 & 17218.4 & 31 & 4 & 2.2707 \\
12 & 11553 & 11438.1 & 32 & 0 & 1.4224 \\
13 & 7573 & 7540.84 & 33 & 1 & 0.890119 \\
14 & 5024 & 4939.65 & 34 & 0 & 0.556496 \\
15 & 3221 & 3217.98 & 35 & 2 & 0.347608 \\
16 & 1828 & 2086.41 & 36 & 0 & 0.216946 \\
17 & 1213 & 1347.11 & 37 & 0 & 0.135291 \\
18 & 788 & 866.585 & 38 & 0 & 0.0843062 \\
19 & 572 & 555.646 & 39 & 0 & 0.0524979 \\
20 & 362 & 355.233 & 40 & 0 & 0.0326686 \\
\hline \hline
\end{tabular}

Table 1. Observed events $(D)$ and expected events $(B)$, used for demonstration.

from which we immediately find the $p$-value for the excess:

$$
p \text {-value }=P\left(k \geq k_{\text {obs }}\right)=I_{\varepsilon}\left(k_{\text {obs }}, n-k_{\text {obs }}-1\right), \quad k_{\text {obs }}>n \varepsilon
$$

which is defined for $k_{\text {obs }}<n$ (in case $k_{\text {obs }}=n$ one has $\left.P(k \geq n)=P(k=n)=\operatorname{Bi}(n \mid n, \varepsilon)=\varepsilon^{n}\right)$.

\subsection{Unfolded data}

If the histogram is the product of some unfolding procedure, things are much less clear, because the bin populations do not follow a well-known and generally applicable probability distribution. In addition, the correlations across adjacent bins are unavoidable. It is unclear even if one should try to compute, bin by bin, the significance of the difference between unfolded data and theoretical expectation. This could mislead people to think that the bins are independent, which is not true. For this reason, this document does not deal further with unfolded spectra.

\section{Some ways to present deviations in Poisson distributed data}

The goal of this section is to present a logical progression of plotting options, which will lead to the final proposal. We examine the different options in detail because they continue to be used despite many people know their drawbacks. All the examples will use the observed (from Monte Carlo simulation) and expected event counts of Table 1 and assume Poisson-distributed bin populations, which is the most common case in high-energy physics (Sec. 1.3 explains the treatment of binomial data). Two additional features have been introduced in the observed data, an excess and a deficit, to make the illustration more interesting. In case the data follow neither a Poisson nor a binomial distribution, one needs to compute the $p$-value according to the probability distribution which the data are assumed to follow. Besides the different $p$-value definition, the rest of this discussion still applies.

\subsection{The $D / B$ ratio}

A simple, and very common practice (e.g., Fig. 13 in Ref. [6] and Fig. 1 in Ref. [7]), is to plot the ratio of data over expectation $(D / B)$, or subtract 1 to plot their relative difference, i.e. $(D-B) / B$. As shown in Fig. 2, clearly this is the worst way to compare two histograms, especially when the bin contents span several orders of magnitude. It is possible to hide significant discrepancies, as it will become obvious.

The biggest disadvantage of this method is that it does not show the statistical significance. It gives the impression that low-population bins are fluctuating more significantly than high-population bins, which is not true in this case. 


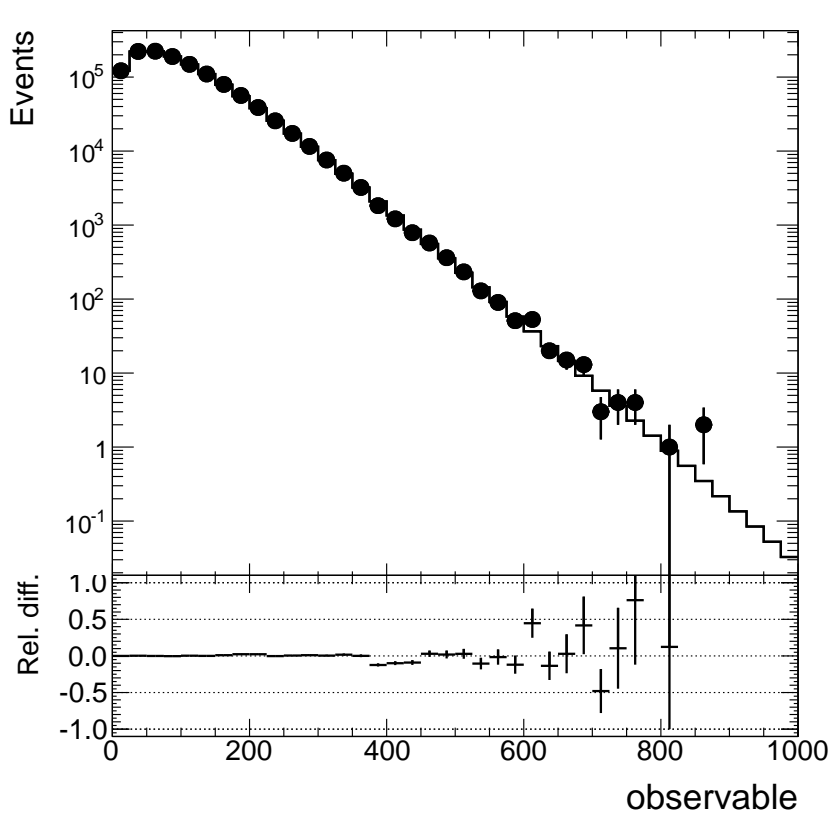

Fig. 2. The relative difference between data $(D)$ and expectation $(B):(D-B) / B$ is not a good indicator of the statistical significance of the observed deviations.

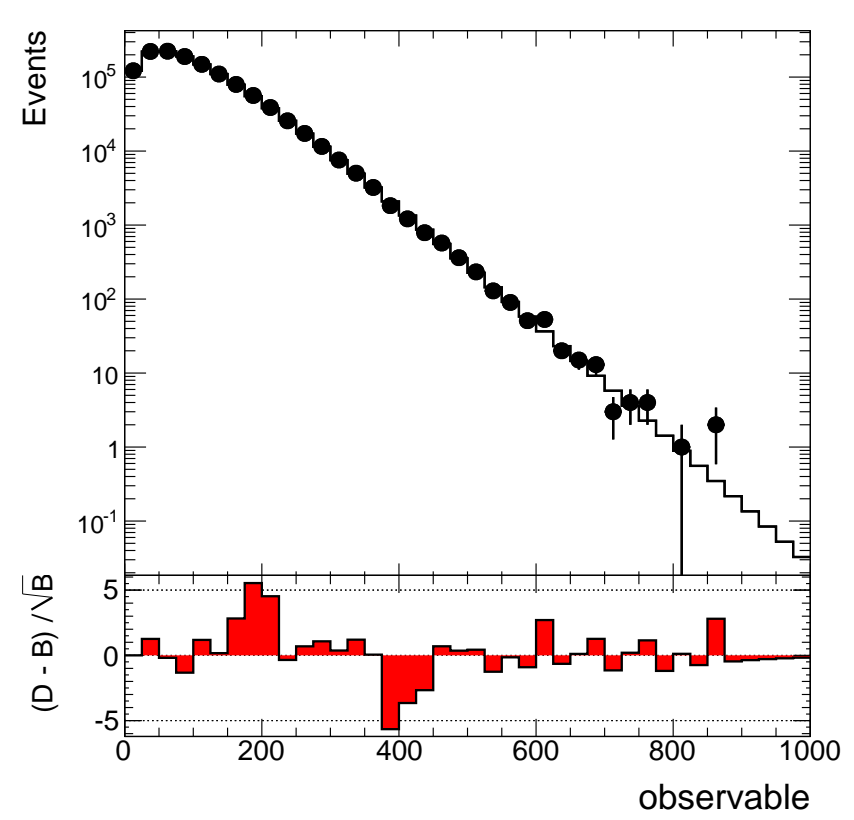

Fig. 3. The quantity $(D-B) / \sqrt{B}$ approximates the statistical significance in bins with large population. However, this approximation fails when there are only a few entries.

The last non-empty bin is off scale, and if we un-zoom the vertical axis to include it, then the other features will become even harder to see. Although the relative difference cannot be smaller than -1 (for $D=0$ ), it has no upper limit, therefore, the absolute values of negative and positive deviations are not comparable. Bins with $D=0$ would all be at relative difference -1 , regardless of $B$, and are typically not shown.

The only advantage of this method is that excesses of data appear above 0 , and deficits below, which is intuitive.

Although this method is inappropriate to convey the statistical significance of deviations, it might still be used when a statistical comparison is not desired. For example, one may wish to show simply that the data lie within $5 \%$ from the expectation, even if a $5 \%$ deviation would be statistically significant in regions with many events and insignificant in regions with just a few.

\subsection{The $(D-B) / \sqrt{B}$ approximation}

It is well-known that, for large $B$, the Poisson distribution with parameter $B$ is approximated well by a Gaussian of mean $B$ and standard deviation $\sqrt{B}$. This leads to the approximation of the Poisson $z$-value with $\frac{D-B}{\sqrt{B}}$, which is plotted in Fig. 3. For example, Ref. [8] contains such a plot.

An advantage of this approach is that we see clearly two significant features: an excess around 200 and a deficit around 400. Intuitively, excesses point "up" and deficits "down". Indeed, the quantity shown, in bins with large expectation, is a good approximation of the actual Poisson $z$-value.

The main disadvantage is that, in low-population bins, $\frac{D-B}{\sqrt{B}}$ is not a good approximation of the true $z$-value. We will see soon that the last bin with $D=2$ is not as significant as it appears in Fig. 3. Similarly, the empty bins in the end of the spectrum do not actually have deficits of $z$-value between 0 and 1 , as is indicated by the small negative bars. Such $z$-values would correspond to $p$-values $<0.5$, but, as we are about to show, these bins contain deficits of $p$-value $>0.5$, which the approximation in Fig. 3 fails to describe.

\subsection{Plotting signed $z$-values}

Instead of approximating the $z$-value with $\frac{D-B}{\sqrt{B}}$, it would be better to plot the exact $z$-value in each bin. If a bin contains a significant excess of data (with $p$-value $<0.5$ ), it will have a $z$-value $>0$ which, when plotted, will give correctly the impression of an excess. If a bin contains a significant deficit of data (with $p$-value $<0.5$ ), it will have a $z$-value $>0$ as well, so, to avoid giving the impression of an excess in bins with deficits, instead of $z$-value we plot $-z$-value in these bins. The result is in Fig. 4 . 


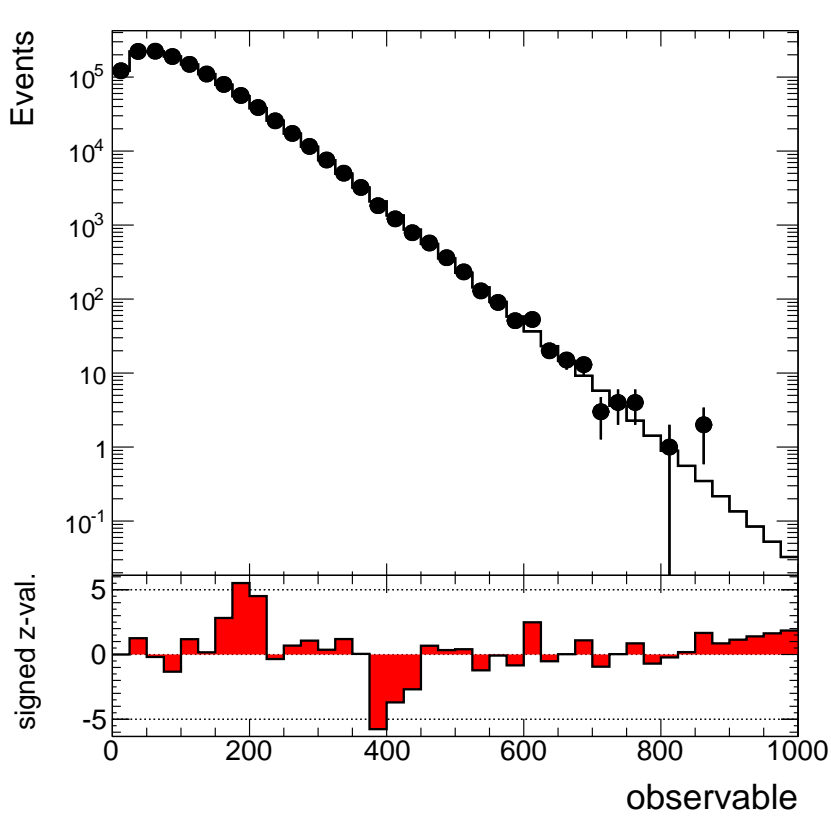

Fig. 4. Showing the $z$-value corresponding to the $p$-value in each bin. In bins with $D<B$, the $-z$-value is plotted, to distinguish excesses from deficits.

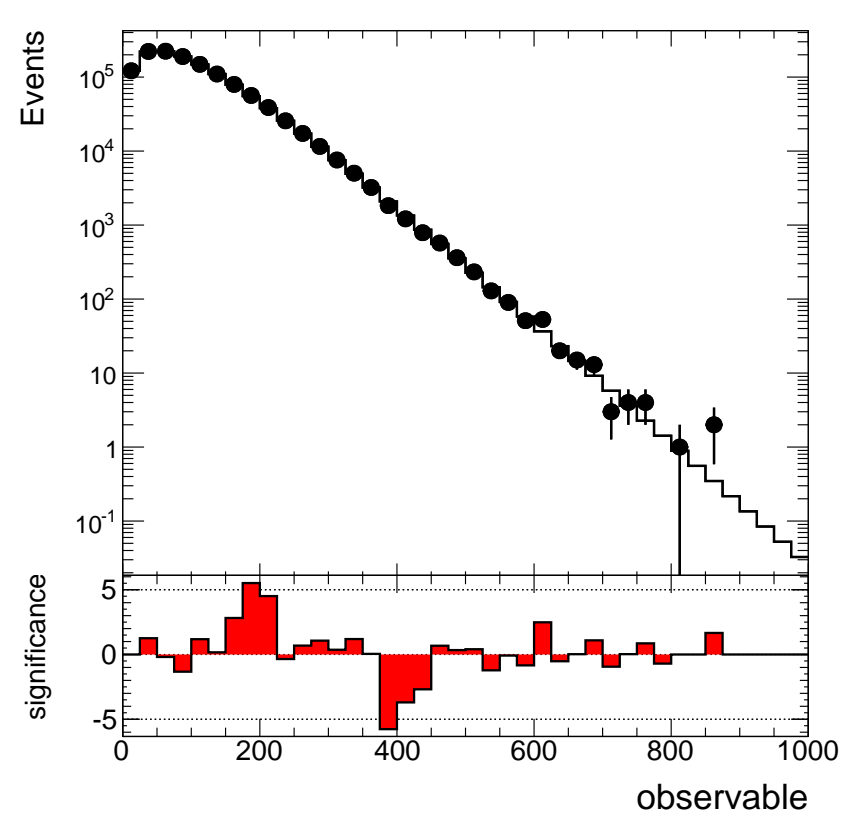

Fig. 5. The final proposal. Plotting the $z$-value only for bins with $p$-value $<0.5$, with positive sign for excesses and negative for deficits.

This is a more accurate representation of significance than $\frac{D-B}{\sqrt{B}}$, but there is still something confusing in bins with low statistics. We know that the last few bins contain deficits $(D=0)$, however, they appear in Fig. 4 as if they were excesses. This happens because they originally had negative $z$-values, indicating very insignificant deficits, so, with the sign-flipping they appear like excesses. The less significant the deficit, the larger the $p$-value, and the more negative the $z$-value, which gives the impression of an even larger excess when plotting $-z$-value. The same problem, but in the opposite direction, is observed in bin 33, around 800. It contains a very insignificant excess with $z$-value $<0$, and since it is an excess this $z$-value is plotted without changing sign. The result points "down", giving wrongly the impression of a deficit.

\subsection{The final proposal: plot signed $z$-values only if $p$-value $<0.5$}

In conclusion, our proposal is to plot $z$-values as in section 2.3 , which are negative in bins with data deficits, with the exception of bins with $p$-value $>0.5$, whose significance should be simply not shown. The result is shown in Fig. 5 .

The justification for not showing the significance of bins where $p$-value $>0.5$ is that such bins agree perfectly with expectation: such deviations are totally uninteresting. In addition, trying to present their $z$-values could be misleading, as explained in section 2.3 .

The advantages of this proposal are the following:

- The $z$-value is accurate, unlike the $(D-B) / \sqrt{B}$ approximation.

- Positive values represent excesses of data over the expectation, and negative values correspond to deficits.

- No feature is hidden if it is worth showing, namely if it has a $p$-value $<0.5$.

- Bins with high and low statistics (like bin 35 with $D=2$ and bin 32 with $D=0$ ) are treated in the same way.

- If the significance is not shown for a bin, it means that there is no relevant deviation from the expectation.

- What we propose is easy to implement, using existing ROOT functions as we show in sections 1.1, 1.2 and 1.3.

\section{Including the theoretical uncertainty}

Any theoretical uncertainty in the reference value will affect the significance of the observation. For example, in case of a Poisson process with parameter $B$, a non-zero uncertainty on $B$ will decrease the significance of the difference between $D$ and $B$. Because realistic use cases always involve some (perhaps small) theoretical uncertainty, it is important to study its effects on our estimate of the significance of the result. A full treatment would require to solve an inference 


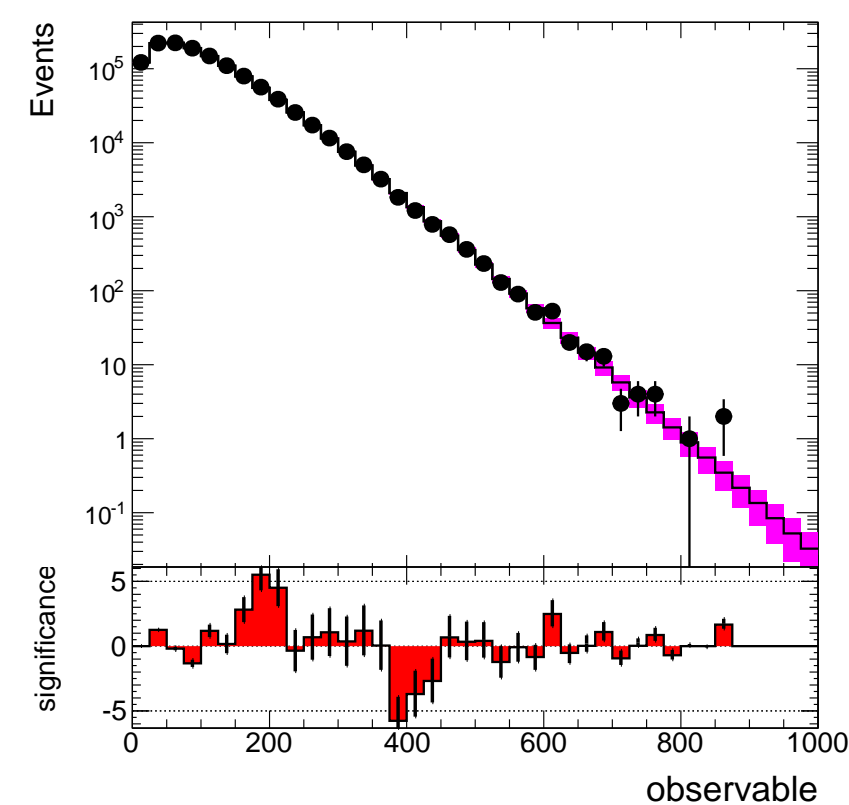

Fig. 6. The error bars in the inset indicate the range by which the significance varies when recomputed after shifting the expectation by \pm 1 standard deviation, corresponding to the error boxes shown in the main plot. This is not the correct way of accounting for theoretical uncertainties.

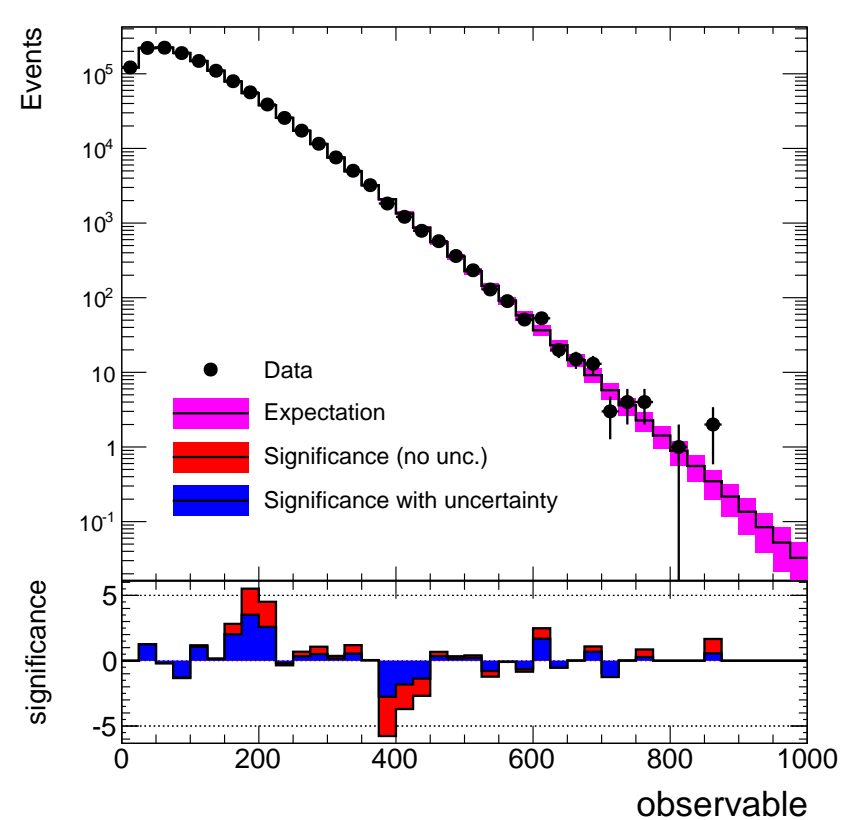

Fig. 7. The inset shows the significance computed by neglecting the theoretical uncertainty on the expectation (red histogram) and by including it (blue histogram). Including additional sources of uncertainty decreases the significance.

problem. However, here we limit ourselves to the aspects which are relevant when making plots, and consider only the total theoretical uncertainty, without addressing all possible components which may contribute to it.

Of course, in some cases it makes sense not to show the effect of theoretical uncertainties. For example, this can be done if the theoretical uncertainty is negligible compared to the statistical uncertainty, or if the deviations without theoretical uncertainty are already not significant enough to deserve further investigation. On the other hand, before claiming than an excess is really statistical significant one should always check what happens when including the total uncertainty on the expectation.

At first sight, a simple procedure to warn the reader about the effects of the theoretical uncertainty on $B$ would seem to plot the significance shown in Fig. 5 with "error bars". The upper and lower deviations with respect to the significance computed above could correspond to the result of recomputing the significance with the parameter $B$ shifted by \pm 1 standard deviation (assuming symmetric uncertainty). An example of this is in Fig. 6 , where the relative uncertainty in bin $i$ has been set to $10^{-5} \cdot i^{3}$. The problem with this approach is that it misses the fundamental point: any additional uncertainty will decrease the significance of the observed deviation.

This can be shown with a formal approach, which would correspond to a Bayesian treatment of the uncertainties. It consists of computing the $p$-value with the marginal model obtained after integration over the parameter, whose prior describes the experimenter's degree of belief on the allowed range of values. This is explained in detail in the next two sections for the Poisson and binomial models, and it is illustrated by Fig. 7, which should be compared to Fig. 6.

\subsection{Poisson model with uncertain parameter}

In any bin the probability of observing $n$ events is given by the Poisson distribution with parameter $Y>0$ (the expected number of events), which is not certain. One assumes to know its best estimate (which we take to be the expectation $B$ of $Y$ ) and "uncertainty" (which we take to be the square root $S$ of the variance of $Y$ ). In most cases $B$ and $S$ are the result of some auxiliary measurement. We choose to model the uncertainty on the Poisson paramter with a function belonging to the Gamma family, which is the conjugate family for the Poisson model. This allows to perform the integration analytically. A unique Gamma density corresponds to the pair $(B, S)$. If we write the Gamma density as

$$
\operatorname{Ga}(x \mid a, b)=\frac{b^{a}}{\Gamma(a)} x^{a-1} e^{-b x}
$$


the expectation and variance are

$$
E=\frac{a}{b}=B \quad \text { and } \quad V=\frac{a}{b^{2}}=S^{2}
$$

from which we obtain the Gamma parameters

$$
a=\frac{B^{2}}{S^{2}} \quad \text { and } \quad b=\frac{B}{S^{2}}
$$

In order to evaluate the probability of observing $n$ events, when the expected yield is described by the Gamma density above, we make use of the marginal model which is obtained by integrating over all possible values of $Y$. The result is the Poisson-Gamma mixture (also known as negative binomial)

$$
P(n \mid a, b)=\int_{0}^{\infty} \operatorname{Poi}(n \mid Y) \operatorname{Ga}(Y \mid a, b) \mathrm{d} Y=\frac{b^{a}}{\Gamma(a)} \frac{\Gamma(n+a)}{n !(1+b)^{n+a}}
$$

(the Gamma function is available in ROOT as $\Gamma(x)=$ ROOT : :Math: : tgamma (x)). We now proceed as above, by defining the $p$-value and $z$-value ${ }^{1}$ in terms of the marginal model (13):

$$
p \text {-value }= \begin{cases}P\left(n \geq n_{\text {obs }}\right)=1-\sum_{n=0}^{n_{\text {obs }}-1} P(n \mid a, b)=\int_{z \text {-value }}^{\infty} \mathcal{N}(x ; 0,1) \mathrm{d} x & , n_{\text {obs }}>B \\ P\left(n \leq n_{\text {obs }}\right)=\sum_{n=0}^{n_{\text {obs }}} P(n \mid a, b)=\int_{-\infty}^{z \text {-value }} \mathcal{N}(x ; 0,1) \mathrm{d} x & , n_{\text {obs }} \leq B\end{cases}
$$

(some care needs to be taken when summing over the marginal model (13): it is recommended to make use of the recursive relations which allow to compute the $n$-th term starting from the term $n-1)$. Finally, we plot the $z$-value only if $p \leq 0.5$ as explained above, to obtain the results shown in Fig. 7 .

Often the theoretical uncertainty is modeled with a Gaussian distribution, instead of the Gamma density used here. Provided that the part of the Gaussian which extends to negative (unphysical) values is negligible, one obtains a result (by numerical integration) which is practically identical to what we obtained above (analytically) using Gamma. However, in cases where the negative Gaussian tail is non-negligible, the Gaussian needs to be truncated at zero. This makes its mean parameter different from its actual mean, and its width parameter different from its actual standard deviation. In addition, the truncated Gaussian gives non-null probability to the value of zero (see Ref. [10] for additional comments). For these reasons, a truncated Gaussian is a poor choice for the probability model. It is highly recommended to use a density function with the correct domain, as the Gamma density, which has the additional advantage of making the integration possible analytically.

\subsection{Binomial model with uncertain parameter}

Let the observed ratio be $k_{\text {obs }} / n$ in a bin for which we expect a binomial distribution with parameter $\varepsilon \in[0,1]$ which is uncertain. We choose to model our knowledge about $\varepsilon$ by means of a Beta distribution with known parameters $a, b$. The Beta functions constitute the conjugate family for the binomial model [9], which allows us to perform the integration analytically. The parameters $a, b$ can be found by with the method of moments or numerically. We assume that they have been determined in order to best represent the result of some auxiliary measurement. Then the probability of observing $k$ events passing the selection out of the initial $n$ events is given by the marginal model

$$
P(k \mid n, a, b)=\int_{0}^{1}\left(\begin{array}{l}
n \\
k
\end{array}\right) x^{k}(1-x)^{n-k} \frac{x^{a-1}(1-x)^{b-1}}{B(a, b)} \mathrm{d} x=\frac{\left(\begin{array}{l}
n \\
k
\end{array}\right)}{B(a, b)} \int_{0}^{1} x^{k+a-1}(1-x)^{n-k+b-1} \mathrm{~d} x
$$

where the last integral is the definition of the Euler's Beta function $B(k+a, n-k+b)$. Hence

$$
P(k \mid n, a, b)=\left(\begin{array}{l}
n \\
k
\end{array}\right) \frac{B(k+a, n-k+b)}{B(a, b)}
$$

In ROOT, the Beta function $B(x, y)=\Gamma(x) \Gamma(y) / \Gamma(x+y)$ is available as

$$
B(x, y)=\text { ROOT : :Math : : } \operatorname{beta}(\mathrm{x}, \mathrm{y})
$$

1 Defined as in eq. 14 and 16 , the $z$-value is automatically negative for deficits. 
which makes the marginal model (15) easy to compute, although summing over it is better achieved if the recurrence relations which connect $P(k \mid n, a, b)$ to $P(k-1 \mid n, a, b)$ are exploited.

The $p$-value and $z$-value are then defined in terms of the marginal model (15) as

$$
p \text {-value }= \begin{cases}P\left(k \geq k_{\text {obs }}\right)=\sum_{k=k_{\text {obs }}}^{n} P(k \mid n, a, b)=\int_{z \text {-value }}^{\infty} \mathcal{N}(x ; 0,1) \mathrm{d} x \quad, k_{\text {obs }} \geq n \varepsilon \\ P\left(k \leq k_{\text {obs }}\right)=\sum_{k=0}^{k_{\text {obs }}} P(k \mid n, a, b)=\int_{-\infty}^{z \text {-value }} \mathcal{N}(x ; 0,1) \mathrm{d} x \quad, k_{\text {obs }}<n \varepsilon\end{cases}
$$

Finally, one plots only the $z$-values which correspond to $p \geq 0.5$ as explained in the previous sections.

Similarly to the Poisson case, the choice of a Gaussian distribution to model the theoretical uncertainty is problematic, because in the binomial case the domain is limited to the interval $[0,1]$, so the Gaussian may have to be truncated on both sides. The Beta density is strongly recommended because it has the correct domain and it allows for analytic integration. In cases where the Gaussian distribution does not span much below 0 or above 1 , it is practically indistinguishable from the Beta distribution with the same mean and standard deviation.

\section{Summary}

In this article, we propose an improved way of plotting the difference between data and expectation, based on the significance of the difference in each bin. We have shown than one can obtain an accurate plot of the statistical significance of the deviation of the bin contents from the expectation, obtaining at the same time an intuitive picture of the relevant deficits and excesses. This is achieved by computing the exact $p$-value and, when its value is smaller that $50 \%$ probability, by mapping it into the $z$-value which gives the deviation in units of Gaussian standard deviations. The sign of $z$-values is always positive for excesses and negative for deficits, making it trivial to decode the result at first sight.

When there are important deviations, whose $z$-values are above 3 or 4 , it is fundamental to check what happens by including the total uncertainty on the expectation. This always ${ }^{2}$ lowers the actual significance and provides a result which is closer to what one would have obtained by performing a proper statistical inference. Here the focus is only on methods which can improve illustrations. However, it is important to notice that our formulae for the $p$-values in the presence of uncertainty on the expectation coincide with the solutions $p_{\Gamma}$ for the Poisson case and $p_{\mathrm{Bi}}$ for the binomial case, computed by Cousins, Linnemann, and Tucker [10] using a completely different approach. These authors have performed frequentist evaluations of the performance of such estimators and concluded that they are optimal for statistical inference. We repeat that we do not focus on statistical inference here, but this agreement between completely independent derivations makes us more confident about the validity of our proposal.

\section{Acknowledgements}

We thank Alex Read, Glen Cowan, and Kyle Cranmer for their feedback.

\section{References}

1. I. Antcheva et al., "ROOT: A C++ framework for petabyte data storage, statistical analysis and visualization", Comput. Phys. Commun. 180 (2009) 2499-2512, doi: 10.1016/j.cpc.2009.08.005.

2. D. Casadei, "Statistical methods used in ATLAS for exclusion and discovery", proceed. of PHYSTAT2011, CERN, 17-20 Jan 2011, arXiv: 1108.2288 .

3. The ATLAS Collaboration, "Search for New Physics in the Dijet Mass Distribution using $1 \mathrm{fb}^{-1}$ of pp Collision Data at $\sqrt{s}=7 \mathrm{TeV}$ collected by the ATLAS Detector", subm. to Phys. Lett. B, arXiv: 1108.6311.

4. M. Abramowitz and I.A. Stegun (eds.), Handbook of Mathematical Functions with Formulas, Graphs, and Mathematical Tables, Dover, New York, 1965, ISBN 978-0486612720.

\footnotetext{
2 After publication we realized that this statement is not always true. When the significance without extra uncertainties is large, then indeed adding extra uncertainty lowers the significance. But if the significance is small without considering additional uncertainties, it is actually possible that it increases when additional uncertainties are convoluted. This is a wellknown effect which can be explained, qualitatively, in this way: If without extra uncertainties the data agree (almost) perfectly with expectation, then convoluting alternative possibilities could make the agreement worse, since these alternatives cannot possibly entail better than perfect agreement.
} 
5. The ATLAS Collaboration, "Search for New Physics in Dijet Mass and Angular Distributions in pp Collisions at $\sqrt{s}=7 \mathrm{TeV}$ Measured with the ATLAS Detector", New J. Phys. 13 (2011) 053044, doi: 10.1088/1367-2630/13/5/053044, arXiv: 1103.3864 .

6. The ATLAS Collaboration, "Measurement of inclusive jet and dijet cross sections in proton-proton collisions at 7 TeV centre-of-mass energy with the ATLAS detector", Eur. Phys. J. C 71 (2011) 1512, arXiv: 1009.5908.

7. The CDF Collaboration, "Search for new particles decaying into dijets in proton-antiproton collisions at $\sqrt{s}=1.96$ TeV", Phys.Rev.D79:112002,2009, arXiv: 0812.4036.

8. The ATLAS Collaboration, "Search for New Physics in Dijet Mass and Angular Distributions in pp Collisions at $\sqrt{s}=7$ TeV Measured with the ATLAS Detector", New J.Phys.13:053044,2011, arXiv: 1103.3864.

9. D. Casadei, "Estimating the selection efficiency", arXiv: 0908.0130.

10. R.D. Cousins, J.T. Linnemann, \& J. Tucker, "Evaluation of three methods for calculating statistical significance when incorporating a systematic uncertainty into a test of the background-only hypothesis for a Poisson process", NIM A 595 (2008) 480-501, doi: 10.1016/j.nima.2008.07.086, arXiv: physics/0702156. 